\title{
FATORES DE RISCO PARA \\ AMPUTAÇÃO MAIOR EM \\ PACIENTES PORTADORES \\ DE PÉ DIABÉTICO
}

\author{
RISK FACTORS FOR MAJOR \\ AMPUTATION IN PATIENTS \\ WITH DIABETIC FOOT
}

PR - BRASIL

\author{
Wagner de Cesare* \\ Marcelo Derbli Schafranski* \\ Ana Luiza Glauser Fontes*** \\ Ricardo Zanetti Gomes ${ }^{* * * *}$
}

\section{RESUMO}

O pé diabético está entre as complicações crônicas do Diabetes mellitus, estando relacionado com altas taxas de amputação de membros inferiores. Dentre as especialidades médicas, uma das que mais destacam-se no estudo dessa enfermidade encontra-se a cirurgia vascular. Sabendo disso, a Universidade Estadual de Ponta Grossa conta com um projeto de extensão denominado "Acompanhamento dos pacientes do ambulatório de angiologia e cirurgia vascular", que visa diversas atividades acadêmicas e sociais. Inúmeras linhas de pesquisa são provenientes deste projeto extensionista, sendo uma dessas pesquisas totalmente voltada para o pé diabético. A confecção deste artigo foi feita exclusivamente com dados obtidos do projeto de extensão, cujo objetivo é identificar fatores de risco para amputação maior não traumática em pacientes portadores de pé diabético. Trata-se de um estudo de coorte, entre $2012 \mathrm{e}$ 2015, estando incluídos 43 pacientes internados por pé diabético. A primeira coleta de dados foi realizada através de um exame clínico, incluindo dados sociodemográfios. A segunda coleta, para registrar o desfecho (amputação maior), foi realizada via telefone. Os fatores de risco avaliados foram diversos, entre os quais estão amputação menor prévia, arteriopatia e hipertensão arterial sistêmica. Não foi encontrado valor estatisticamente significativo para os fatores de risco avaliados. Concluiu-se que os fatores de risco estudados não apresentaram associação com o desfecho amputação maior.

Palavras-chave: pé diabético; amputação; fatores de risco.

\footnotetext{
* Aluno de graduação da Universidade Estadual de Ponta Grossa (UEPG), PR - Brasil. E-mail: wagnerdecesare@hotmail.com

** Professor da Universidade Estadual de Ponta Grossa (UEPG), PR - Brasil. E-mail: marceloschafranski@yahoo.com.br

*** Aluna de graduação da Universidade Estadual de Ponta Grossa (UEPG), PR - Brasil. E-mail: ana_fontes1@hotmail.com

**** Professor da Universidade Estadual de Ponta Grossa (UEPG), PR - Brasil. E-mail: zanetticons@uol.com.br
} 


\section{ABSTRACT}

Diabetic foot is one of the chronic complications of diabetes mellitus, and it is also related to the high rates of lower limbs amputation. Among the medical specialties, vascular surgery is responsible for most of the studies about the study of diabetic foot. The State University of Ponta Grossa develops an outreach project called "Follow-up of patients from the angiology and vascular surgery ambulatory", which carries out numerous academic and social activities. Numerous research lines are part of this outreach project, one of these studies focuses only on diabetic foot. This article was written exclusively with data obtained from this outreach project that aims to identify risk factors for non-traumatic major amputation in patients with diabetic foot. It was a cohort study carried out from 2012 to 2015 involving 43 patients hospitalized for diabetic foot. The first data collection was done through a clinical examination, including sociodemographic data, whereas the second data collection to record the outcome of major amputations was done via telephone calls. The risk factors assessed were diverse; among them are previous minor amputation, arteriopathy and systemic arterial hypertension. No statistically significant value was found for the risk factors evaluated. It was concluded that the risk factors studied were not associated with the greater amputation outcome.

Keywords: diabetic foot; amputation; risk factors.

\section{Introduçáo}

Diabetes mellitus (DM) é definido como um distúrbio metabólico amplo, sendo a sua principal característica a hiperglicemia. Em estudos epidemiológicos, encontramos que, em 1985, estimava-se cerca de 30 milhôes de casos de diabetes mellitus em adultos no mundo, crescendo para 173 milhóes em 2002, com estimativa de 300 milhóes para 2030 (ARAÚJO, 2013-2014; ANGELO et al., 2010). $\mathrm{O}$ diabetes mellitus atinge $7,6 \%$ da população brasileira entre 30 e 69 anos e aproximadamente $20 \%$ da população acima dos 69 anos. Segundo a International Diabetes Federation (IDF), a quarta ou quinta principal causa básica de morte na maioria dos países desenvolvidos é o diabetes mellitus; além disso, aparece como epidemia nos países em desenvolvimento (ALMEIDA, 2008).

Caso o acompanhamento e/ou tratamento sejam inadequados, o diabetes mellitus evolui para complicaçóes crônicas que acabam por limitar a qualidade de vida do paciente e comprometer sua sobrevida (ALMEIDA, 2008). Em se tratando das complicaçóes crônicas do diabetes mellitus, o pé diabético é uma das mais frequentes, com altas taxas de amputação e internamentos hospitalares prolongados. Grande parte desses internamentos são associados a infecçôes. Pacientes diabéticos apresentam de 15 a 46 vezes maior risco para amputaçáo de membros inferiores do que pacientes com glicemia normal, sendo que $1,7 \%$ dos internamentos tem como motivo amputação (SANTOS et al., 2011). Nos Estados Unidos, aproximadamente $50 \%$ dos casos de amputação não traumáticas de membros inferiores são devido ao diabetes mellitus (BRASILEIRO et al., 2005).

O pé diabético é definido como neuropatia periférica associada ou não à doença vascular periférica, desencadeada por traumatismo prévio, produzindo ulceração do pé (DUARTE, 2011). Classifica-se o pé diabético em dois tipos: o neuropático e o neuro-isquêmico, acometendo $65 \%$ e $35 \%$ dos casos, respectivamente (DUARTE, 2011). As úlceras diabéticas, em 70 a 100\% dos casos, ocorrem de maneira secundária à neuropatia, associadas a variados graus de doença vascular. Além disso, amputaçóes prévias de membros inferiores, doença arterial periférica (DAP), tabagismo, etilismo, dislipidemia, hipertensão arterial sistêmica (HAS) e tempo de diabetes mellitus, são considerados fatores de risco ou agravantes para ulceraçōes (ALMEIDA, 2008; PORCIÚNCULA et al., 2007; BORTOLETTO et al., 2007).

As amputaçóes de membros inferiores são classificadas em maiores e menores. As maiores são descritas como aquelas que ocorrem proximais ao pé, como desarticulaçáo do quadril, amputação 
transfemoral e amputação transtibial, enquanto as menores representam amputaçôes dos dedos dos pés e a parte anterior o pé (transmetatarsiana) (SPICHLER, 2004; ASSUMPÇÃO et al., 2009; MIYAJIMA et al., 2006).

O objetivo deste trabalho foi identificar fatores de risco para amputação maior não traumática em pacientes portadores de pé diabético em um serviço de cirurgia vascular periférica, na cidade de Ponta Grossa

\section{Materiais e métodos}

\section{Tipo de estudo}

Trata-se de um estudo observacional do tipo coorte, com amostra inicial de 62 pacientes. Essa amostra inicial foi composta por pacientes internados por motivo do pé diabético, entre fevereiro de 2012 e janeiro de 2015, em algum dos seguintes hospitais: Santa Casa de Misericórdia de Ponta Grossa, Hospital Bom Jesus e Hospital Universitário Regional dos Campos Gerais, independente da sua classificação, em isquêmico, infeccioso ou neuropático.

Todo o arsenal de dados utilizados para a realizaçáo deste trabalho foi obtido através do projeto de extensão que visa atividades acadêmicas e sociais, intitulado "Acompanhamento dos pacientes do ambulatório de angiologia e cirurgia vascular", da Universidade Estadual de Ponta Grossa, vinculado ao curso de Medicina.

\section{Critérios de inclusão e exclusão}

Foram incluídos no estudo todos os pacientes que compóem a amostra inicial, desde que concordassem em participar. Os critérios de exclusão utilizados foram: (1) pacientes que não possuíam contato disponível por telefone; (2) que não apresentavam dados suficientes em ficha e/ou que não souberam fornecer as informaçóes necessárias; e (3) que possuíam amputação maior prévia. O estudo foi aprovado pelo Comitê de Ética em Pesquisa em Seres Humanos (COEP) da Universidade Estadual de Ponta Grossa, sob o parecer de número 903.495. Todos os indivíduos envolvidos assinaram o Termo de Consentimento Livre e Esclarecido (TCLE).

\section{Amostragem e coleta de dados}

A primeira coleta foi realizada através de um formulário desenvolvido pelos pesquisadores, o qual incluía dados sociodemográficos (gênero, idade e escolaridade) e clínicos (amputação prévia, arteriopatia, realização de cirurgia cardíaca, dislipidemia, etilismo, hipertensão arterial sistêmica, neuropatia periférica, tabagismo, tempo de diabetes mellitus e realizaçáo de terapia renal substitutiva (TRS)). Essa primeira coleta de dados se deu entre 8 de fevereiro de 2012 e 30 de novembro de 2014. Na segunda coleta realizada (entre 5 de janeiro de 2014 e 5 de janeiro de 2015), os pacientes foram contatados por telefone para registro do desfecho (amputação maior ou não amputação maior). $\mathrm{O}$ tempo de seguimento entre as duas coletas foi data em média \pm desvio padrão. Em caso de mais 
de uma amputaçáo posterior à primeira coleta, foi considerada a anatomicamente mais proximal. Dos 62 pacientes da amostra total, 43 (69,3\%) foram incluídos no estudo.

\section{Fatores de Risco}

Os fatores de risco avaliados foram amputaçấo menor prévia, arteriopatia, cirurgia cardíaca, escolaridade sem ensino superior, dislipidemia, etilismo, hipertensão arterial sistêmica, neuropatia periférica, tabagismo, tempo de diabetes mellitus superior a 10 anos e TRS.

A técnica do exame neurológico dos pés com o monofilamento de SemmesWeinstein (MSW) de 10g, para sensibilidade tátil, foi realizada em 10 pontos distribuídos pela regiáo plantar dos pés, sendo 1 para cada pododáctilo, 3 pontos para os metatarsos (cabeças do primeiro, terceiro e quinto metatarsos), 1 no mediopé e outro no calcanhar. Em cada um desses 10 pontos, foi realizada uma pressáo com o monofilamento levemente curvado, com duração de 1 a 2 segundos, realizado duas vezes e sem a visualização do exame pelo paciente.

Para a sensibilidade vibratória, utilizamos um diapasão de $128 \mathrm{~Hz}$ em posição pendular à pele, na cabeça do primeiro metatarso e no maléolo medial, sendo o exame realizado duas vezes e sem a visualizaçáo do paciente, comparada posteriormente com a do processo estiloide do rádio em uma das mãos. Foram considerados pacientes neuropatas aqueles com quaisquer alteraçôes no exame neurológico proposto (hipoestesia, anestesia, hipoapalestesia e apalestesia).

A arteriopatia foi avaliada com a utilizaçáo de um doppler vascular portátil, sendo analisados os pulsos pedioso e tibial posterior de ambos os membros. A partir do padráo de fluxo, definimos os arteriopatas aqueles com padrão bifásico, monofásico ou ausência de fluxo.

Pacientes já operados para cirurgia cardíaca, com escolaridade sem ensino superior, dislipidêmicos, etilistas, hipertensos, tabagistas, com tempo de diabetes mellitus maior do que 10 anos e em realização atual ou prévia de TRS, foram assim denominados por autointitulação durante anamnese para preenchimento da ficha clínica.

\section{Desfecho}

Definiu-se desfecho como quadro clínico com evolução para amputação maior. Dessa maneira, o agrupamento de todos os pacientes com tal evoluçáo nominou-se de grupo "amputação maior" (AM). Todos os outros pacientes deram origem ao grupo "não amputação maior" (NAM), ou seja, aqueles que não necessitaram de amputação maior. Amputaçáo maior e menor foram definidas de acordo com seus níveis, previamente descritos, podendo ocorrer em qualquer momento entre a primeira e segunda coleta.

\section{Análise Estatística}

A amostra foi testada para distribuição normal (teste de Shapiro-Wilk). Os dados sáo apresentados em valores percentuais, média e desvio-padráo. Para a comparaçáo de 
variáveis quantitativas, foi utilizado o teste t de Student bicaudal. Para comparação de variáveis categóricas, foi realizado o teste exato de Fisher bicaudal. Em relaçáo aos valores de $p$, foram considerados estatisticamente significativos valores $<0,05$. Toda a avaliação estatística foi realizada por meio do programa MedCalc versão 12.4.0 (Ostend, Bélgica).

\section{Resultados}

De nossa amostra total (43 pacientes), 11 (25,6\%) indivíduos compóem o grupo AM e $32(74,4 \%)$ o grupo NAM. Ambos os grupos (AM vs NAM) não diferiram de maneira estatisticamente significativa em relação a idade, sexo e tempo de seguimento (Tabela 1).

Tabela 1. Características de base

\begin{tabular}{|c|c|c|c|c|}
\hline & AM & NAM & TOTAL & $\mathrm{p}$ \\
\hline & $\mathrm{n}=11$ & $\mathrm{n}=32$ & $\mathrm{n}=43$ & \\
\hline \multicolumn{5}{|l|}{ Gênero } \\
\hline Masculino & $9(81,8 \%)$ & $21(65,6 \%)$ & $30(69,8 \%)$ & \\
\hline Feminino & $2(18,2 \%)$ & $13(34,4 \%)$ & $13(30,2 \%)$ & 0,45 \\
\hline Idade (anos)* & $61,3 \pm 9$ & $65,5 \pm 12,3$ & $64,4 \pm 11,6$ & 0,30 \\
\hline Tempo de Seguimento (dias)* & $587,8 \pm 268$ & $597,9 \pm 342,1$ & $595,3 \pm 321,8$ & 0,92 \\
\hline
\end{tabular}

A média de idade da amostra foi de 64,4 $\pm 11,6$ anos, sendo que os grupos AM e NAM obtiveram valores bastante semelhantes entre si e com a amostra total $(61,3 \pm 9$ e $65,5 \pm 12,3$, respectivamente). O sexo masculino foi predominante em nosso estudo (30/69,8\%), sendo maioria em ambos os grupos, AM (9/81,8\%) e NAM (21/65,6\%). Considerando a amostra total de pacientes, a média de tempo de seguimento foi de $595,3 \pm 321,8$ dias (587,8 \pm 268 no grupo AM e 597,9 $\pm 342,1$ no grupo NAM)

A classificação quanto ao nível de escolaridade revelou escolaridade sem ensino superior em 90,9\% (10 indivíduos) do grupo AM e 93,7\% (30 indivíduos) do grupo NAM, representando $93,0 \%$ do grupo como um todo.

Em relação ao fator de risco amputaçáo menor prévia, dos 43 pacientes da amostra total, 14 (32,5\%) apresentavam esse quadro (Tabela 2). Desses, 35,7\% (5/14) evoluíram para o grupo AM e 64,3\% (9/14) evoluíram para o grupo NAM. Não obtivemos diferença estatisticamente significativa do fator de risco amputaçáo prévia para o desfecho amputação maior $(\mathrm{p}=0,45)$. 
Tabela 2. Fatores de risco analisados para amputaçáo maior

\begin{tabular}{l|l|l|l|l}
\hline & $\mathrm{AM}$ & $\mathrm{NAM}$ & TOTAL & $\mathrm{p}^{*}$ \\
\cline { 2 - 5 } & $\mathrm{n}=11$ & $\mathrm{n}=32$ & $\mathrm{n}=43$ & \\
\hline Amputaçáo Prévia & $5(45,4 \%)$ & $9(28,1 \%)$ & $14(32,5 \%)$ & 0,45 \\
\hline Arteriopatia & $11(100 \%)$ & $29(90,6 \%)$ & $40(93,0 \%)$ & 0,55 \\
\hline Escolaridade sem ensino superior & $10(90,9 \%)$ & $30(93,7 \%)$ & $40(93,0 \%)$ & 1 \\
\hline Cirurgia Cardíaca & $1(9,1 \%)$ & $2(6,2 \%)$ & $3(6,9 \%)$ & 1 \\
\hline Dislipidemia & $6(54,5 \%)$ & $15(46,9 \%)$ & $21(48,8 \%)$ & 0,73 \\
\hline Etilismo & $2(18,2 \%)$ & $12(37,5 \%)$ & $14(32,5 \%)$ & 0,29 \\
\hline HAS & $9(81,8 \%)$ & $23(71,9 \%)$ & $32(74,4 \%)$ & 0,69 \\
\hline Neuropatia & $11(100,0 \%)$ & $31(96,9 \%)$ & $42(97,7 \%)$ & 1 \\
\hline Tabagismo & $4(36,4 \%)$ & $13(40,6 \%)$ & $17(39,5 \%)$ & 1 \\
\hline Tempo de DM > 10 anos & $8(72,7 \%)$ & $22(68,7 \%)$ & $30(69,8 \%)$ & 1 \\
\hline Terapia Renal Substitutiva & $3(27,3 \%)$ & $2(6,2 \%)$ & $5(11,6 \%)$ & 0,09 \\
\hline AM: Dos & & & &
\end{tabular}

AM: Desfecho "Amputação Maior" NAM: Desfecho "Não amputação Maior"

* Teste exato de Fisher

A neuropatia foi observada em 11 (100\%) pacientes do grupo AM e em 31 $(96,9 \%)$ do grupo NAM, ocupando $97,7 \%$ da amostra total. Em relação à arteriopatia, a mesma foi diagnosticada em 9 (100\%) indivíduos do grupo AM e em 29 (90,6\%) indivíduos do grupo NAM, ocupando $93,0 \%$ da amostra total. Não obtivemos diferença estatisticamente significativa para neuropatia $(\mathrm{p}=1)$ e arteriopatia $(\mathrm{p}=0,55)$ para ambos.

Cinco pacientes $(11,6 \%)$ da amostra total se encontravam em TRS, sendo o percentual de dialíticos no grupo AM (3/27,3\%), cerca de quatro vezes o observado no grupo NAM $(3 / 6,2 \%)$, mas sem significância estatística $(\mathrm{p}=0,09)$. Do mesmo modo, a presença de cirurgia cardíaca prévia náo representou um fator de risco para amputação maior $(\mathrm{p}=1)$.

A presença de diabetes mellitus por mais de 10 anos, hábito do tabagismo e etilismo, dislipidemia e hipertensão arterial sistêmica não representaram fatores de risco para amputação maior nesta amostra de pacientes. Com exceção de um paciente diabético do tipo I, toda a amostra era composta por diabéticos do tipo II.

\section{Discussáo}

No presente estudo, analisamos múltiplos fatores de risco para amputação maior em pacientes portadores de pé diabético. Muitos deles já estudados por outros autores (ALMEIDA, 2008; PORCIÚNCULA et al., 2007; BORTOLETTO et al., 2007).

Em relação ao sexo $(\mathrm{p}=0,45)$, nossa amostra foi bastante semelhante àquela analisada por outros pesquisadores. Tavares e colaboradores (2009) avaliaram pacientes internados por pé diabético submetidos a amputaçáo maior, encontrando, de um total de 141 pacientes, $83(58,9 \%)$ indivíduos do sexo masculino e $58(41,1 \%)$ do sexo feminino $(\mathrm{p}=0,073)$ (TAVARES, 2009).

Em estudo realizado por Assumpção e colaboradores (2009), observou-se média de idade de 58,5 $\pm 11,4$ anos para 93 pacientes com pé diabético avaliados quanto a fatores de risco para amputação maior e menor. Em estudo de Prado dos Santos e colaboradores 
(2006), obteve-se média de idade de 60,2 $\pm 12,4$ anos para 99 pacientes portadores de pé diabético; desses, 51 pacientes $(51,5 \%)$ sofreram amputaçáo maior (SANTOS et al., 2006). Em nossa amostra, a idade média de pacientes com amputação maior observada foi maior $(64,4 \pm 11,6)$.

Em relaçáo ao nível de escolaridade, também não encontramos diferença estatisticamente significativa. Em desacordo a isso, análise de 64 pacientes portadores de pé diabético, por Santos e colaboradores (2011), concluiu que a baixa escolaridade (0-4 anos de estudo) é um fator de risco importante para amputação maior ( $\mathrm{OR}=1,9 ; \mathrm{p}=0,04)$. Por outro lado, em trabalho prévio desse mesmo autor (2008), pacientes que cursaram ensino básico (fundamental e médio, completo ou incompleto) correspondiam a maior prevalência entre os portadores de pé diabético, mesmo assim, não obtiveram valores estatisticamente significativos ( $\mathrm{p}=0,86)$ (SANTOS, 2006).

Em nosso estudo, daqueles pacientes com amputação prévia, 35,7\% delesevoluíram para nova amputação maior. Em um trabalho realizado por Nunes e colaboradores (2006), de 80 pacientes diabéticos internados por úlceras nos pés, 22\% (18 indivíduos) apresentavam histórico de amputação prévia $(\mathrm{p}=0,11)$. Desses, $72 \%$ (13) realizaram novo procedimento, embora tenham sido tanto amputaçóes maiores como menores (NUNES et al., 2006). Tais dados corroboram com nossos achados, uma vez que ambos os trabalhos não resultaram em valores estatisticamente significativos. Entretanto, em estudo de Real Collado e colaboradores (2001), obteve-se taxa de amputaçáo de 9,2\% (14/152) entre portadores de pé diabético, sendo que, destes, $50 \%$ possuíam amputaçóes prévias $(\mathrm{p}=0,036)$, poucos evoluíram para amputação maior $(5 / 14)$.

Segundo nossos resultados, pressupor que pacientes submetidos à amputaçáo menor prévia irão, em algum momento, evoluir para uma amputação maior, não se mostrou verdadeiro. Sempre que possível, amputaçôes menores devem ser indicadas, uma vez que não estão associadas a amputação maior futura $(\mathrm{p}=0,45)$.

Em um estudo realizado em 2006, não foi encontrada significância estatística para o tempo de diabetes mellitus relacionado à amputação de membros inferiores (MIYAJIMA et al., 2006). Nosso estudo se mostrou semelhante, obtendo valores náo estatisticamente significativos. Consideramos a relação de amputaçáo maior apenas para tempo de diabetes mellitus maior do que 10 anos.

Almeida (2008), em sua tese de mestrado, analisou 124 pacientes submetidos à amputação não traumática de membros inferiores, desses, 66 indivíduos (53\%) eram diabéticos, entre os quais 60\% (39/66) tinham diagnóstico de diabetes mellitus há mais de 10 anos. Nesse mesmo trabalho, o autor reafirma insignificância para amputaçóes prévias.

É sabido que a arteriopatia presente em pacientes diabéticos leva a um risco 17 vezes maior de evolução para gangrena do que em não diabéticos (BORTOLETTO et al., 2007). Corroborando com este achado, apesar de não termos encontrando associaçáo estatisticamente significativa, nosso estudo contou com elevada prevalência de arteriopatiaem ambos os grupos (AM e NAM). Entretanto, em um estudo de Miyajima e colaboradores (2006), com amostra de 210 pacientes com lesóes em pé diabético, realizouse amputação maior 45 indivíduos (21,4\%), destes, 44 (98\%) apresentavam arteriopatia. Com realização de arteriografia, observou-se que 88,7\% (39/44) apresentavam múltiplas estenoses $(\mathrm{p}=0,0015)$, constituindo arteriopatia como um fator de risco importante para amputação maior (MIYAJIMA et al., 2006). Diferentemente do encontrado em nosso estudo. 
Porciúncula e colaboradores (2007), analisando 32 indivíduos diabéticos neuropatas, observaram úlceras nos pés, prévias ou presentes, em 18 (50\%) pacientes. Da amostra total, hipertensão arterial sistêmica $(81,2 \%)$ e dislipidemia $(81,2 \%)$ ocuparam o mesmo percentual. A relaçáo com amputaçáo de membros náo foi definida nesse estudo; porém, a relação com ulceração dos pés não foi significativa para ambos, hipertensão arterial sistêmica $(\mathrm{p}=0,36)$ e dislipidemia $(\mathrm{p}=0,67)$.

Em outro estudo, encontramos resultados semelhantes em se tratando da correlação entre hipertensão arterial sistêmica e amputação de membros inferiores, sendo que também nấo se obteve resultado estatisticamente significativo $(\mathrm{p}=0,63)$ (ASSUMPÇÃ̄O et al., 2009). Do mesmo modo, em nossa amostra não houve associação entre hipertensão arterial sistêmica e dislipidemia com amputaçáo maior.

A presença de cirurgia cardíaca em nossos pacientes foi de $6,9 \%$ (3 indivíduos). Obtivemos 9,1\% (1 indivíduo) de cirurgias no grupo AM e 6,2\% (2 indivíduos) no grupo NAM. Não foi encontrada significância estatística. A revisão da literatura mostrouse pobre em estudos envolvendo pé diabético e cirurgia cardíaca. Entretanto, fica claro, em outros trabalhos, que a doença arterial coronariana está frequentemente associada ao diabetes mellitus, sendo, inclusive, mais grave nesses pacientes do que naqueles não diabéticos ${ }^{17,18,19}$.

No estudo de Miyajima e colaboradores (2006), aqui já citado, foram avaliados os desfechos amputação maior, amputação menor e não amputação, sendo que a presença de nefropatia esteve em todos os grupos. Aqueles pacientes com nefropatia em hemodiálise obtiveram número significativamente maior no grupo de amputação maior $(\mathrm{p}=0.0051)$. Concluiu-se que hemodiálise é fator de risco independente para amputaçáo maior. Em nossa amostra, 27,3\% (3 indivíduos) dos pacientes do grupo AM e 6,2\% (2 indivíduos) do grupo NAM eram nefropatas em TRS. Não obtivemos significância estatística.

Em um estudo observacional de coorte desenvolvido por Armstrong e colaboradores (2014), foram avaliados 204 pacientes tabagistas com claudicação ou isquemia crítica. Em um curso de 1 ano, 61 deles cessaram o hábito de fumar e mantiveram-se em abstinência. No grupo dos não tabagistas 38\% (23/61) eram diabéticos, enquanto que, entre os tabagistas, $40 \%$ (57/143). Após um seguimento de 5 anos, os pacientes que pararam de fumar apresentaram, em relação ao grupo de fumantes, menor mortalidade $(14 \%$ vs. $31 \%)$ e melhor sobrevida livre de amputação $(81 \% \text { vs } 60 \%)^{20}$. Não obtivemos significância estatística para o tabagismo em nosso estudo.

Assim como o tabagismo, o etilismo é conhecido por favorecer as complicaçóes do diabetes mellitus. Pacientes que ingerem bebida alcoólica frequentemente acabam por desenvolver neuropatia sensitiva periférica em sete vezes maior risco daqueles que faziam uso moderado de bebidas alcoólicas (BORTOLETTO et al., 2007). Não encontramos correlação entre o etilismo e amputação maior em nossa amostra $(\mathrm{p}=0,29)$.

A neuropatia diabética em nossa amostra não representou um fator de risco para amputação maior, uma vez que ambos os grupos, AM e NAM, apresentaram taxas acima de $90 \%$ para neuropatia. Este dado está em acordo com trabalho realizado por Assumpçáo e colaboradores (2009), no qual também náo obtiveram significância estatística $(\mathrm{p}=0,117)$ (ASSUMPÇÃO et al., 2009). Por outro lado, Santos e colaboradores (2013) observaram que 59,8\% (64/107) dos pacientes submetidos a amputaçáo apresentavam algum grau de neuropatia, contra $37,4 \%(40 / 107)$ no grupo controle $(\mathrm{p}=0,002)$, sugerindo um papel importante da neuropatia para amputação maior. 
As principais limitaçôes do nosso estudo foram: (i) impossibilidade de análise da totalidade dos pacientes propostos por dados incompletos ou falta de contato, de 62 pacientes existentes, apenas 43 pacientes foram abordados, o que pode resultar em um viés de amostragem; (ii) os dados não significativos podem ser resultantes de uma amostra reduzida, portanto, com baixo poder; (iii) a determinaçáo dos fatores de risco nem sempre seguiu o padrão ouro para o seu diagnóstico, como é o caso da hipertensão arterial sistêmica e dislipidemia, onde os pacientes que se autodenominavam como portadores dos mesmos, assim foram tratados na análise.

Através do presente estudo, concluímos que nenhum dos fatores de risco estudados apresentou valor estatisticamente significativo para amputação maior.

\section{Referências}

ALMEIDA, E.C.G. Diabetes Mellitus como causa de amputação não traumática no hospital de clínicas da Universidade Federal de Uberlândia. Dissertação (Mestrado em Ciências da Saúde). 2008. Disponível em: http://www.ceatenf.ufc.br/Artigos/26. pdf.

ANGELO. B.Z. et al. Fatores preditores de mortalidade em pacientes diabéticos em terapia de substituição renal em Tubarão-SC. Arq Catar Med., v.39, p. 23-8, 2010.

ARAÚJO, Silva A. Diretrizes da Sociedade Brasileira de Diabetes. Sociedade Brasileira de Diabetes - SBD. Barueri: Farmacêutica; 2013-2014.

ARMSTRONG, E.J.; WU, J.; SINGH, G.D.; DAWSON, D.L.; PEVEC, W.C.; AMSTERDAM, E.A.; LAIRD, J.R. Smoking cessation is associated with decreased mortality and improved amputation-free survival among patients with symptomatic peripheral artery disease. J VascSurg., v.60, n.6, p. 1565-71, 2014.

ASSUMPÇÃO, E.C. et al. Comparação dos fatores de risco para amputações maiores e menores em pacientes diabéticos de um Programa de Saúde da Família. J Vasc Bras., v.4, n. 8, p.133-38, 2009.

BECKLES, G.L.; CHOU, C.F. Diabetes - United States, 2006 and 2010. MMWR SurveillSumm., v.62, n.3, p. 99-0, 2013.

BORTOLETTO, M.S.S.; VIUDE, D.F.; HADDAD, M.C.L.; KARINO, M.E. Caracterização dos portadores de diabetes submetidos à amputação de membros inferiores em Londrina, Estado do Paraná. Acta. Sci. Health. Sci., v.32, v.2, p.205-13, 2010.

BRASILEIRO, J.L.; OLIVEIRA, W.T.P.; MONTEIRO, L.B.; CHEN, J.; PINHO Jr., E.L.; MOLKENTHIN, S. et al. Pé diabético: aspectos clínicos. J Vasc Bras., v.4, p. 11-21, 2005.

DUARTE, N.; GONÇALVES, A. Pé Diabético. Revista de Angiologia e Cirurgia Vascular, v.7, p. 65-79, 2011. 
KALIL, R.A.K. Cirurgia de revascularização do miocáridio no diabetes mellitus. ArqBrasEndocrinolMetab., v.51, v, 2, p. 345-51, 2007.

LEDUR, P.; ALMEIDA, L.; PELLANDA, L.C.; SCHAAN, B.D. Perfil e evolução dos pacientes com diabetes mellitus submetidos à cirurgia de revascularização miocárdica em serviço de referência no sul do Brasil. RevAssocMedBras, v. 57, v.2, p.200-4, 2011.

MIYAJIMA, S.; SHIRAI, A.; YAMAMOTO, S.; OKADA, N.; MATSUSHITA, T. Risk factors for major limb amputations in diabetic foot gangrene patients. Diabetes Res ClinPract., v. 71, p.272-9, 2006.

NUNES, M.A.P.; RESENDE, K.F.; CASTRO, A.A.; PITTA, G.B.B.; FIGUEIREDO, L.F.P.; MIRANDA, F.J. Fatores predisponentes para amputação de membro inferior em pacientes diabéticos internados com pés ulcerados no estado de Sergipe. J VascBras., v.5, p. 123-30, 2006.

PORCIÚNCULA, M.V.P.; ROLIM, L.C.P.; GAROFOLO, L.; FERREIRA, S.R.G. Análise de fatores associados à ulceração de extremidades em indivíduos diabéticos com neuropatia Periférica. ArqBrasEndocrinolMetabol, v. 51, v. 7, p.1134-42, 2007.

REAL COLLADO, J.T.; VALLS, M. BASANTAALARIO, M.L.; AMPUDIABLASCO, F.J.; ASCASOGIMILIO, J.F.; CARMENA RODRÍGUEZ, R. Estudio de factoresasociadosconamputación, en pacientes diabéticos conulceraciónen pie. An. Med. Interna (Madrid) [revista eletrônica]. 2001. Disponível em: http:// scielo.isciii.es/scielo.php?script=sci_arttext\&pid=S0212-71992001000200002\&lng=es.

SANTOS, I.C.R.V.; SOBREIRA, C.M.M.; NUNES, E.N.S.; MORAIS, M.C.A. Prevalência e fatores associados a amputações por pé diabético. Ciênc. saúde coletiva, v. 18, n. 10, p. 3007-14, 2013.

SANTOS, I.C.R.V.; SOUZA, W.V.; CARVALHO, E.F.; MEDEIROS, M.C.W.C.; NÓBREGA, M.G.L.; LIMA, P.M.S. Prevalência de pé diabético e fatores associados nas unidades de saúde da família da cidade do Recife, Pernambuco, Brasil, em 2005. Cad. Saúde Pública, Rio de Janeiro, v.24, n.12, p. 2861-70, 2008.

SANTOS, I.C.R.V.; NUNES, E.N.S.; MELO, C.A.; FARIAS, D.G. Amputações por pé diabético e fatores sociais: implicações para cuidados preventivos de enfermagem. Rev Rene, Fortaleza, v. 12, v. 4, p. 684-91, 2012.

SANTOS, V.P.; SILVEIRA, D.R.; CAFFARO, R.A. Risk factors for primary major amputation in diabetic patients. Med J., São Paulo, v.124, n 2, p. 66-70, 2006.

SPICHLER, E.R.S.; MIRANDA Jr., F.; SPICHLER, E.S.; FRANCO L.J. Amputações maiores de membros inferiores por doenca arterial periférica e diabetes melito no municipio de Rio de Janeiro. J VascBras, v.3, n.2, p.111-22, 2004.

TAVARES, C.M.S. et al. Perfil de clientes submetidos à amputações relacionadas ao diabetes mellitus. Rev Bras Enferm., v.62, n. 6, p. 825-30, 2009. 